\title{
Patterns of Care for Radiotherapy in the Neoadjuvant and Adjuvant Treatment of Gastric Cancer: A Twelve-Year Nationwide Cohort Study in Korea
}

\author{
Jee Suk Chang, MD ${ }^{1}$ \\ Young Choi, BA,3 \\ Jaeyong Shin, MD ${ }^{34}$ \\ Kyung Hwan Kim, MD1,5 \\ Ki Chang Keum, MD, PhD' \\ Hyo Song Kim, MD, PhD 6 \\ Woong Sub Koom, MD, PhD' \\ Eun-Cheol Park, MD, PhD 3,4
}

\section{${ }^{1}$ Department of Radiation Oncology, Yonsei Cancer Center, Yonsei University College of Medicine, Seoul, ${ }^{2}$ Department of Public Health, Graduate School, Yonsei University, Seoul, ${ }^{3}$ Institute of Health Services Research, Yonsei University College of Medicine, Seoul, ${ }^{4}$ Department of Preventive Medicine, Yonsei University College of Medicine, Seoul, ${ }^{5}$ Graduate School of Medical Science and Engineering, Korea Advanced Institute of Science and Technology, Daejeon, ${ }^{6}$ Division of Medical Oncology, Department of Internal Medicine, Yonsei Cancer Center, Yonsei University College of Medicine, Seoul, Korea}

Correspondence: Woong Sub Koom, MD, PhD Department of Radiation Oncology,

Yonsei Cancer Center, Yonsei University

College of Medicine, 50 Yonsei-ro,

Seodaemun-gu, Seoul 03722, Korea

Tel: $82-2-2228-8116$

Fax: 82-2-312-9033

E-mail: mdgold@yuhs.ac

Co-correspondence: Eun-Cheol Park, MD, PhD Department of Preventive Medicine,

Yonsei University College of Medicine,

50 Yonsei-ro, Seodaemun-gu, Seoul 03722, Korea Tel: 82-2-2228-1862

Fax: 82-2-392-7734

E-mail: ECPARK@yuhs.ac

Received December 30, 2016

Accepted March 3, 2017

Published Online March 8, 2017

*Jee Suk Chang, Young Choi, and Jaeyong Shin contributed equally to this work.

\section{Purpose}

Although Korea has the highest incidence of gastric cancer worldwide and D2-lymphadenectomies are routinely performed, radiotherapy (RT) practice patterns have not been well studied. Therefore, we examined RT usage trends for neoadjuvant/adjuvant patients and identified factors associated with RT. We also examined survival benefits and net medical cost advantages of adding RT.

\section{Materials and Methods}

Patients diagnosed with gastric cancer who underwent gastrectomy from 2002-2013 were identified using National Health Insurance Service-National Sample Cohort.

\section{Results}

Annually, 30.9 cases per 100,000 population in crude rate underwent gastrectomy in 230 hospitals and 49.8\% received neoadjuvant/adjuvant therapy in 182 hospitals. For neoadjuvant/adjuvant patients, postoperative chemo-RT was administered in $4 \%$ of cases in 26 hospitals. No significant trends regarding treatment type were observed over time. Having undergone RT was inversely associated with being $\geq 60$ years old and having a low income. Having undergone RT was positively related to having a Charlson comorbidity index $\geq 4$, hospital location and hospital volume ( $\geq 2,000$ beds). Significant portions of patients treated with RT in this nation (52\%) were concentrated in one large-volume hospital. Use of RT linked to increased cost of primary treatment, yet not to reduced overall medical expense. RT did not influence both on overall and disease-specific survivals after adjusting for potential confounders $(p>0.05)$.

\section{Conclusion}

RT was uncommonly utilized as adjuvant or neoadjuvant treatment by physicians in Korea. Despite intrinsic drawback in this data, we did not find either survival benefit or net medical cost advantage by adding RT in adjuvant treatment.

\section{Key words}

Stomach neoplasms, Chemoradiotherapy, Adjuvant radiotherapy, Physicians' practice patterns 


\section{Introduction}

Gastric cancer is the third leading cause of death among men and the fifth leading cause of cancer death among women worldwide [1]. Globally, 1,000,000 new cases of gastric cancer are diagnosed annually, and half of these cases occur in East Asia [2]. Korea has reported the highest incidence of gastric cancer, along with Japan, and has an agestandardized incidence of 56.8 cases per 100,000 men, affecting nearly 30,000 patients in 2013 [3]. Complete surgical resection is the primary therapy used, but a substantial number of patients with a more advanced stage disease (e.g., beyond submucosa or metastasis and into the lymph nodes) experience locoregional, peritoneal, or systemic recurrences after receiving curative gastrectomy alone [4]. Clinical trials have been conducted to reduce the risk of recurrence and mortality, but no international consensus concerning the best oncological strategy has been reached $[5,6]$.

The survival benefit of adjuvant chemoradiotherapy (CRT) for curatively resected gastric cancer was established by the Intergroup (INT)-0116 trial in 2001 [7]. However, recent data from ARTIST and CRITICS trials showed that there was no significant survival benefit in the CRT group over chemotherapy alone for D2-resected patients [8,9]. To date, several underpowered studies have propounded an opinion that some selected patients may still benefit from adding radiotherapy (RT) to chemotherapy even after D2 surgery [10]. At the same time, two multi-institutional phase III trials from Korea and Japan demonstrated the merits of adjuvant chemotherapy [11,12].

Given the controversial and limited data available, the role of RT has been heavily criticized for gastric cancer patients undergoing D2 gastrectomy, which has currently become the standard of care in Europe and the United States as well as in East Asia [13,14]. Furthermore, neither patterns of care nor national data for RT specifically have been explored among nonmetastatic gastric cancer patients in Korea. Therefore, the purpose of this study was to examine RT usage trends for neoadjuvant and adjuvant patients in Korea and to estimate the impact of the available evidence on clinical practice using data from a Korean national cohort from 2002 to 2013. Secondarily, we also examined survival benefits and net medical cost advantages of adding RT in neoadjuvant and adjuvant treatment.

\section{Materials and Methods}

All citizens in Korea are obligated to enroll in the singlepayer, national health insurance, and medical aid program administered by the National Health Insurance Corporation. The present study used data from the National Health Insurance Service-National Sample Cohort (NHIS-NSC), which included 1,025,340 representative subjects (approximately $2.2 \%$ of the country's population), who were randomly stratified and selected based on age, sex, insurance type, income, residential region, and individual total medical costs. Rates were calculated and shown per 1,000,000 population. Details of the NHIS-NSC database are available in a previous report [15].

\section{Data source and collection}

The database includes information regarding basic patient demographics, reimbursement for medical services, disease codes according to the International Classification of Diseases, 10th revision, identifiers for the clinic or hospital, medical history, and mortality. This data is then automatically linked to the Korean National Statistical Office where it is obtainable for research. This study which used data from the NHIS-NSC was exempt from institutional review board evaluation; however, the study protocols adhere to the guidelines outlined in the Declaration of Helsinki of 1964 and later versions in accordance with the ethical standards of the responsible committee on human experimentation (institutional and national).

\section{Description of the study cohort}

Patients were eligible for analysis if they were diagnosed with gastric cancer (ICD-10 code, C16), underwent gastrectomy during the study period (2002-2013), and were $>20$ years old at the time of diagnosis. We excluded patients who had another primary cancer or known metastatic disease at the time of diagnosis. In Korea, surgery alone is recommended in patients with early gastric cancer and adjuvant or neoadjuvant therapy is recommended in patients with stage II-III gastric cancer. Treatments administered within 4 months of surgery were considered as adjuvant or neoadjuvant. The last follow-up was conducted in December 2013, and the median follow-up time for patients who were still alive was 5.2 years (range, 0 to 11.9 years).

\section{Statistical analyses}

RT usage in the neoadjuvant and adjuvant setting was the primary end-point. Patients were grouped into 2-year time 
epochs and the percentage of patients who received neoadjuvant or adjuvant RT for each period from 2002 to 2013 were calculated. Univariate and multivariate logistic regression analyses were performed to investigate whether there was an association between receipt of RT and sex, age (20-49, $50-59,60-69$, or $\geq 70$ years), type of surgery (total vs. subtotal gastrectomy), use of lymph node dissection, the Charlson comorbidity index (sum of weights assigned to predeter-

Table 1. Demographic characteristics of gastric cancer patients who underwent gastrectomy with or without neoadjuvant/ adjuvant treatment from 2002 to 2013

\begin{tabular}{|c|c|c|c|c|}
\hline Characteristic & Total & $\begin{array}{l}\text { Neoadjuvant/ } \\
\text { Adjuvant }(-)^{\text {a) }}\end{array}$ & $\begin{array}{l}\text { Neoadjuvant/ } \\
\text { Adjuvant (+)a) }\end{array}$ & p-value \\
\hline \multicolumn{5}{|l|}{ Sex } \\
\hline Male & $2,436(67)$ & $1,209(66)$ & $1,227(67)$ & 0.320 \\
\hline Female & $1,217(33)$ & $625(34)$ & $592(33)$ & \\
\hline \multicolumn{5}{|l|}{ Age (yr) } \\
\hline $20-49$ & $770(21)$ & $384(21)$ & $386(21)$ & 0.008 \\
\hline $50-59$ & $901(25)$ & $442(24)$ & $459(25)$ & \\
\hline $60-69$ & $1,110(30)$ & $529(29)$ & $581(32)$ & \\
\hline $70-89$ & $872(24)$ & $480(26)$ & $392(22)$ & \\
\hline \multicolumn{5}{|l|}{ Surgery type } \\
\hline TG & $822(23)$ & $305(17)$ & $517(28)$ & $<0.001$ \\
\hline STG/PG & $2,831(77)$ & $1,529(83)$ & $1,302(72)$ & \\
\hline \multicolumn{5}{|l|}{ LND use } \\
\hline No & $83(3)$ & $39(2)$ & $44(3)$ & 0.523 \\
\hline Yes & $3,213(97)$ & $1,602(98)$ & $1,610(97)$ & \\
\hline \multicolumn{5}{|c|}{ Charlson comorbidity index } \\
\hline $0-1$ & $2,216(61)$ & $1,077(59)$ & $1,139(63)$ & 0.054 \\
\hline 2 & $702(19)$ & $375(20)$ & $327(18)$ & \\
\hline 3 & $316(9)$ & $172(9)$ & $144(8)$ & \\
\hline$\geq 4$ & $419(11)$ & $211(11)$ & $209(11)$ & \\
\hline \multicolumn{5}{|l|}{ Income } \\
\hline$\leq 20$ percentile & $562(15)$ & $285(16)$ & $277(15)$ & 0.015 \\
\hline 21-80 percentile & $1,917(52)$ & $923(50)$ & $995(55)$ & \\
\hline$\geq 81$ percentile & $1,174(32)$ & $627(34)$ & $547(30)$ & \\
\hline \multicolumn{5}{|l|}{ Health insurance type } \\
\hline NHI & $3,532(97)$ & $1,770(96)$ & $1,761(97)$ & 0.556 \\
\hline Medical aid & $122(3)$ & $64(4)$ & $58(3)$ & \\
\hline \multicolumn{5}{|l|}{ Residential area } \\
\hline Metropolitan & $1,663(46)$ & $804(44)$ & $859(47)$ & 0.035 \\
\hline Non-metropolitan & $1,991(54)$ & $1,031(56)$ & $960(53)$ & \\
\hline \multicolumn{5}{|l|}{ Hospital location } \\
\hline Metropolitan & $3,161(87)$ & $1,567(85)$ & $1,594(88)$ & 0.051 \\
\hline Non-metropolitan & $493(13)$ & 267 (15) & 225 (12) & \\
\hline \multicolumn{5}{|c|}{ Hospital volume (beds) } \\
\hline$<1,000$ & $1,503(41)$ & $738(40)$ & $765(42)$ & 0.265 \\
\hline$\geq 1,000$ & $2,151(59)$ & $1,096(60)$ & $1,054(58)$ & \\
\hline \multicolumn{5}{|l|}{ Disabled status } \\
\hline No & 3,295 (90) & $1,641(89)$ & 1,654 (91) & 0.132 \\
\hline Yes & $358(10)$ & 193 (11) & $165(9)$ & \\
\hline
\end{tabular}

Values are presented as number (\%). TG, total gastrectomy; STG/PG, subtotal gastrectomy / partial gastrectomy; LND, lymph node dissection; NHI, National Health Insurance. ${ }^{\text {a) }}$ Patients who received chemotherapy and / or radiotherapy before and after the surgery within 3 months were considered as (neo-)adjuvant group. 
mined clinical conditions which were collected from in- and out-patient billing data within the diagnosis year; charlson comorbidity index (CCI); 0-1, 2, 3, or $\geq 4$ ) [16], income percentile $(<20,20-79$, or $\geq 80)$, health insurance type, residential and hospital location (metropolitan or not), hospital volume $(<1,000,1,000-1,999$, or $\geq 2,000$ beds), and disability status. Scatter plots were used to examine practice patterns in relation to hospital volume as a function of XY position. A metropolitan location referred to cities with more than $1,000,000$ people.

We also examined the use of preoperative or postoperative chemotherapy among neoadjuvant and adjuvant patients, the overall incidence of perioperative mortality (patients who died within 1 month of surgery), overall survival (OS; from diagnosis to any cause of death), and disease-specific survival (DSS; from diagnosis to gastric cancer-specific death). OS and DSS were estimated using the Kaplan-Meier method. A Cox proportional hazards survival analysis was performed to identify hazard ratios and $95 \%$ confidence intervals (CI). We calculated direct total medical costs as the sum of inpatient and outpatient services based on claim and reimbursement data by medical service providers from 2002 to 2013. Primary treatment and net medical costs were defined and calculated as medical bill expenses claimed by medical service providers from diagnosis to 3 months' postsurgery and to death/last follow-up, respectively. All costs are presented in U.S. dollars, with an exchange rate of 1,103 Korean won to 1 U.S. dollar, which was the annual average exchange rate in 2008. A threshold of $\mathrm{p}=0.05$ was selected for statistical significance. All statistical analyses were performed using SPSS ver. 20.0 (IBM SPSS Statistics, IBM Corp., Armonk, NY).

\section{Results}

\section{Sociodemographic characteristics}

The baseline sociodemographic characteristics of gastric cancer patients who underwent gastrectomy are described in Table 1 (a more detailed explanation is provided in Supplementary Results, S1 Fig.). An analysis of the demographic characteristics by year revealed that patients who were older, had more comorbid diseases, received medical aid, and was disabled were more likely to undergo surgery from nonmetropolitan hospitals at the end of the study period than at the beginning (all $\mathrm{p}<0.05)$ (S2 Table).

\section{Postoperative mortality}

The overall 1-month postoperative mortality rate was $0.6 \%$. Higher mortality rates were observed in patients $>70$ years old $(1.2 \%, \mathrm{p}=0.039)$, those with a CCI $\geq 4(1.6 \%, \mathrm{p}=0.027)$, and those who underwent total gastrectomy $(1.1 \%, \mathrm{p}=0.038)$ (S3 Table). Hospital volume was also significantly associated with mortality rate $(<1,000,1,000-1,999$, and $\geq 2,000 ; 0.8 \%$, $0.7 \%$, and $0.0 \%$, respectively; $\mathrm{p}=0.018$ ), and patients with a low income ( $\leq 20$ th percentile), who received medical aid, or who underwent postoperative chemotherapy had a doubled mortality rate, although this was not statistically significant (all $\mathrm{p}>0.05)$.

\section{Neoadjuvant or adjuvant treatment}

Almost half (49.8\%) of patients who underwent gastrectomy received adjuvant or neoadjuvant chemotherapy. Between 2002 and 2013, the number of patients who received neoadjuvant or adjuvant treatment decreased by $26 \%$ (from $64 \%$ to $38 \%$ ) (S4 Fig.). However, this may be because of decreases in the number of cases of advanced gastric cancer resulting from

Table 2. Trends in neoadjuvant or adjuvant treatment by the group from 2002 to 2013

\begin{tabular}{|c|c|c|c|c|c|}
\hline Year & $\begin{array}{c}\text { Adjuvant } \\
\text { chemotherapy }\end{array}$ & $\begin{array}{l}\text { Adjuvant } \\
\text { chemo-RT }\end{array}$ & $\begin{array}{l}\text { Neoadjuvant } \\
\text { chemotherapy }\end{array}$ & $\begin{array}{l}\text { Neoadjuvant } \\
\text { chemo-RT }\end{array}$ & p-value ${ }^{a}$ \\
\hline $2002-2003$ & 311 (89.1) & $8(2.2)$ & $30(8.7)$ & 0 & 0.078 \\
\hline 2004-2005 & 296 (87.1) & $15(4.3)$ & $29(8.6)$ & 0 & \\
\hline 2006-2007 & $274(87.5)$ & $10(3.1)$ & $29(9.3)$ & 0 & \\
\hline 2008-2009 & 248 (87.9) & $16(5.5)$ & $19(6.6)$ & 0 & \\
\hline $2010-2011$ & $227(83.2)$ & $17(6.1)$ & $25(9.3)$ & $4(1.4)$ & \\
\hline 2012-2013 & 223 (85.4) & $9(3.4)$ & $28(10.8)$ & $1(0.4)$ & \\
\hline Total & $1,580(86.9)$ & $73(4.0)$ & $161(8.8)$ & $5(0.3)$ & \\
\hline
\end{tabular}

Values are presented as number (\%). RT, radiotherapy. ${ }^{a}$ The linear-by-linear association test was used to examine trends over time. 


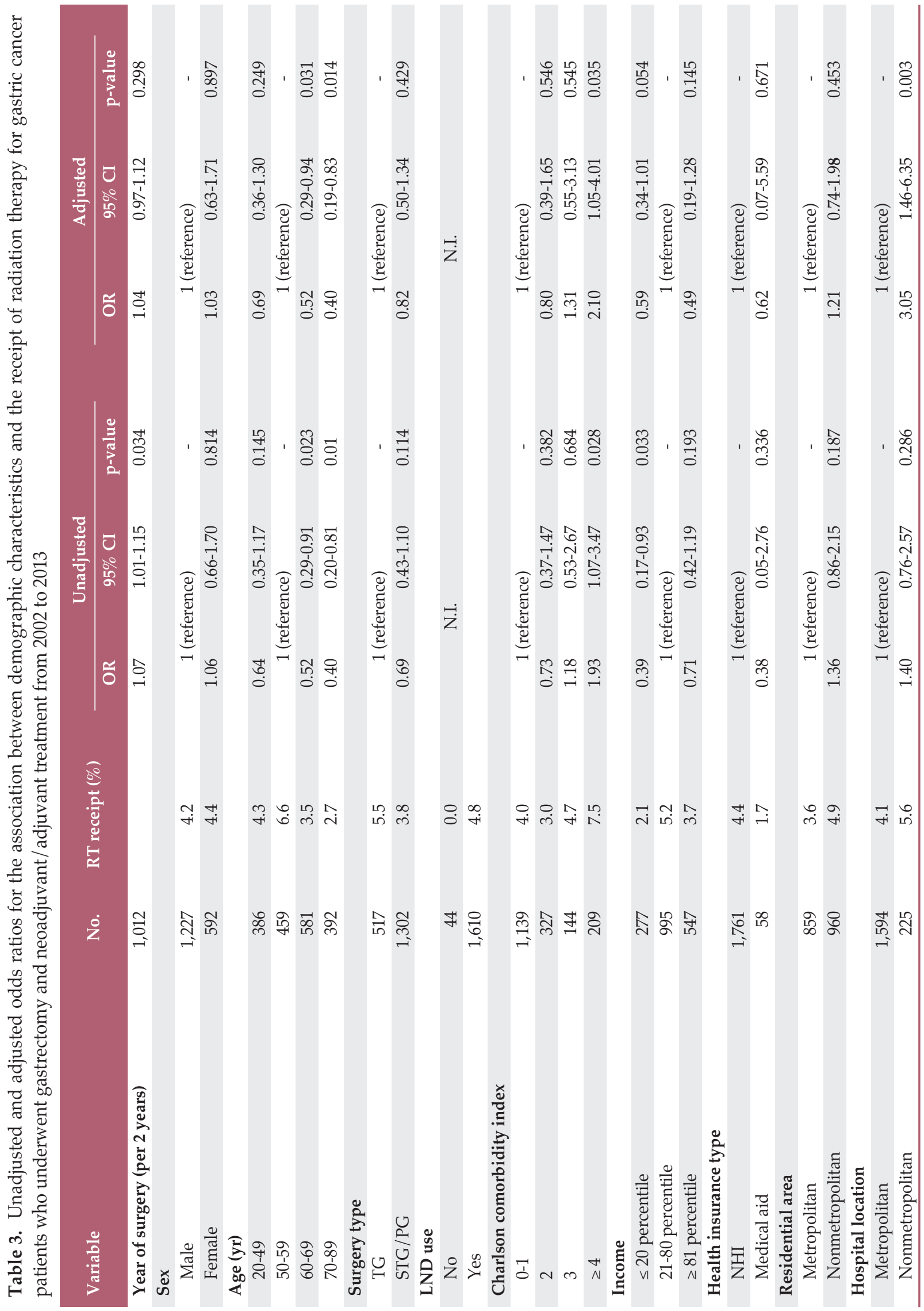




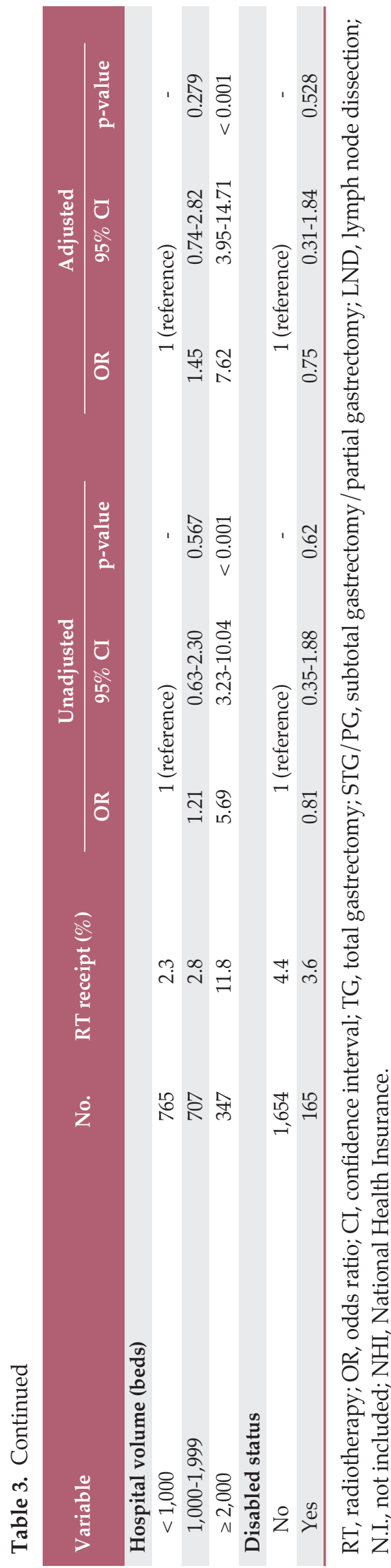

the nationwide gastric cancer screening and surveillance program implemented in 1999 (a more detailed explanation is provided in Supplementary Results) [17]. Also, the neoadjuvant/adjuvant treatment group displayed similar sociodemographic characteristics by year relative to those of entire cohort (S2 and S5 Tables and S6 Fig.).

\section{Patterns of neoadjuvant and adjuvant treatment}

Postoperative adjuvant chemotherapy was administered in most cases (86.9\%), followed by neoadjuvant chemotherapy $(8.8 \%)$, adjuvant CRT (4\%), and neoadjuvant CRT $(0.3 \%)$. The sociodemographic characteristics of patients who received RT did not change significantly by year. No trends were found regarding treatment modality by year among any of the four treatment groups (Table 2). The number of patients receiving neoadjuvant chemotherapy increased (by 4.2\%) from 20082009 to 2012-2013. The opposite trend was noted in the adjuvant chemotherapy and adjuvant CRT groups (from 87.9\% and 5.5\% in 2008-2009 to $85.4 \%$ and 3.4\% in 2012-2013, respectively).

\section{Factors associated with receipt of RT}

Having undergone RT was inversely associated with being $\geq 60$ years old and having a low income ( $\leq 20$ th percentile). Having undergone RT was positively associated with having a CCI $\geq 4$ and hospital location (nonmetropolitan). Hospital volume ( $\geq 2,000$ beds) was found to be the strongest factor affecting the use of RT (vs. $<1,000$ beds; adjusted odds ratio, $7.62 ; 95 \%$ CI, 3.95 to 14.71 ) (Table 3).

Among 230 hospitals, only 26 hospitals (12.1\%) used RT, and the median proportion of RT use in neoadjuvant and adjuvant patients in each hospital was 4.1\% (Fig. 1A). Additionally, one large hospital used RT with chemotherapy $(47.6 \%)$ as often as chemotherapy alone $(47.6 \%)$ in that hospital and seemed to care for a significant portion of the RT patients in the nation $(52 \%)$.

On the other hand, neoadjuvant chemotherapy was used in 74 hospitals (34.6\%), and the median proportion of neoadjuvant chemotherapy use among neoadjuvant/adjuvant patients in each hospital was 7.5\% (Fig. 1B). Adjuvant chemotherapy was used in nearly all hospitals (98.6\%), and an average of $90.9 \%$ of neoadjuvant/adjuvant patients received adjuvant chemotherapy in each hospital (Fig. 1C).

\section{Costs}

The mean cost for primary treatment was $\$ 7,437$ per patient of neoadjuvant/adjuvant treatment. The average net cost from diagnosis to death or last follow-up was $\$ 13,965$ per patient. Costs by various subgroups are presented in Table 4 . Primary 
A

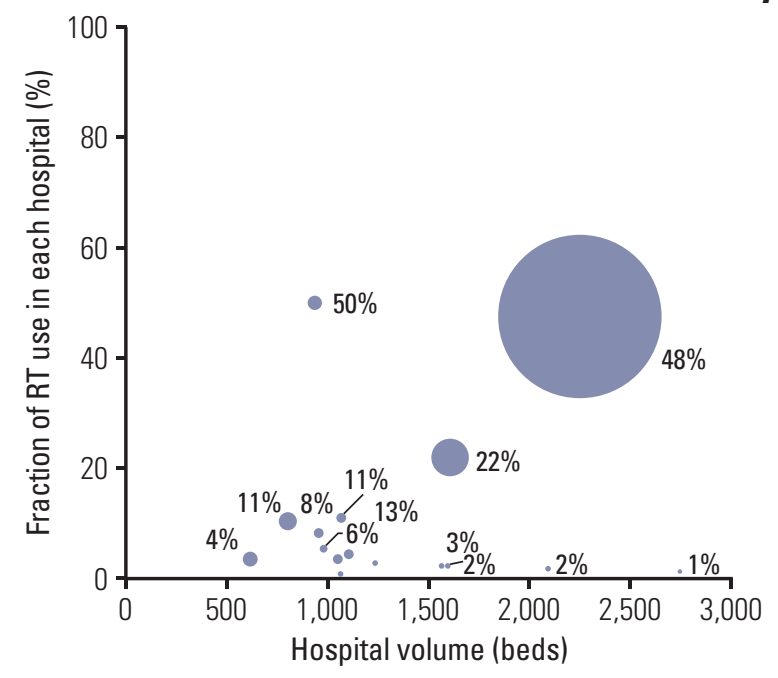

B

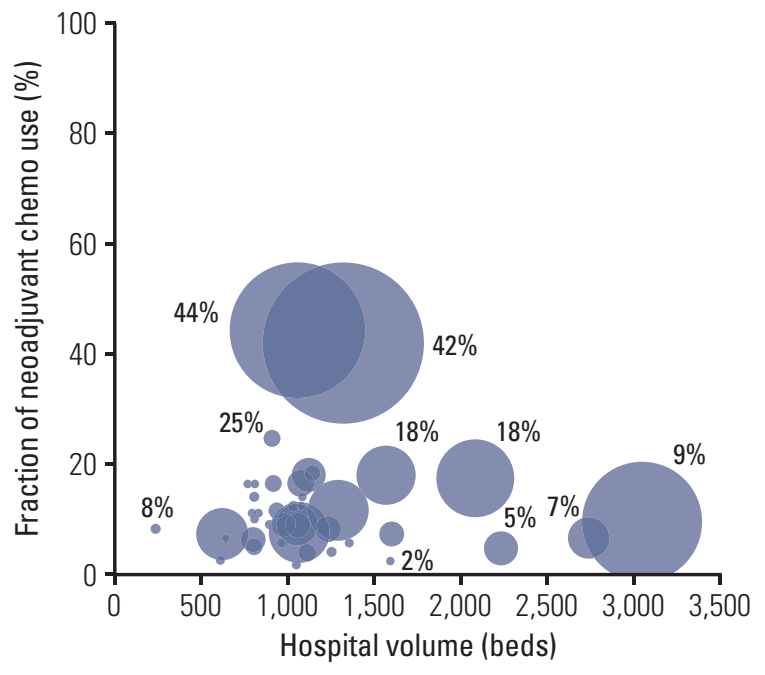

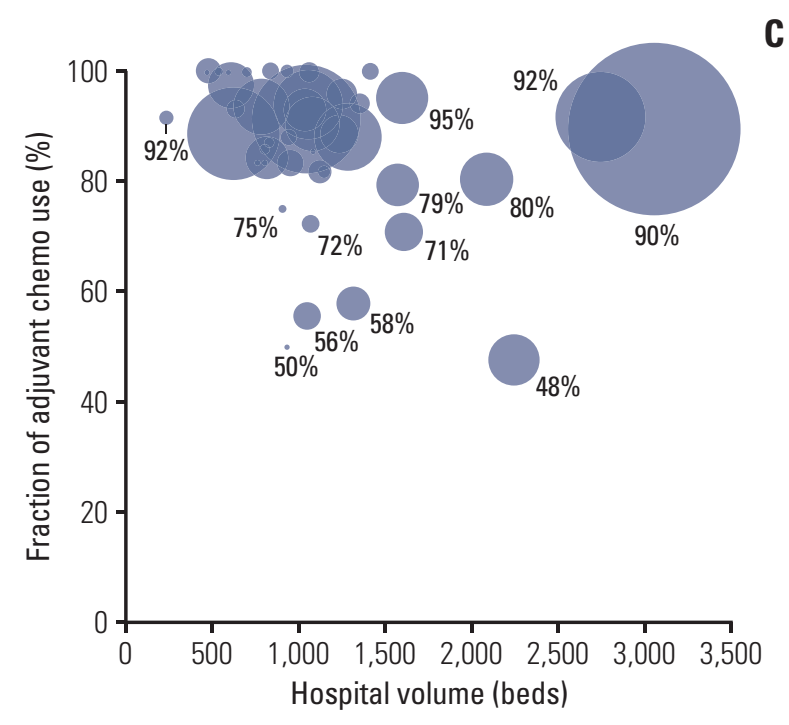

Fig. 1. Bubble charts for the proportion of patients by the hospital. Two-dimensional bubble charts indicate how the proportions of adjuvant chemoradiotherapy (A), neoadjuvant chemotherapy (B), and adjuvant chemotherapy use (C) in each hospital change by hospital volume. The size of the bubble corresponds to the proportion within each treatment group. RT, radiotherapy.

treatment costs were higher for patients with total gastrectomy (vs. subtotal), a higher CCI ( 3 or $\geq 4)$, disabled status, RT, and from a large volume hospital (all $p<0.05)$. Total medical costs were higher for patients with total gastrectomy (vs. subtotal), a higher CCI ( 3 or $\geq 4)$, RT, from a high-income group $(\geq 81$ percentile), and from a large volume hospital (all $\mathrm{p}<0.05$ ). Elderly was found to be related to lower total medical cost. The mean costs of primary treatment were $\$ 3,734$ per person higher for patients who received RT with chemotherapy than those receiving chemotherapy alone. This cost difference was sustained and even more pronounced when it came to net medical cost $(\$ 6,884$ per person).

\section{Survival analyses}

Patients who underwent gastrectomy and received adjuvant or neoadjuvant therapies for gastric cancer in Korea showed a 5-year OS of 66.5\% (95\% CI, 64.2 to 68.9) and a 5-year DSS of 
Table 4. Kaplan-Meier estimated 5-year survival rate and mean cost for gastric cancer patients who underwent gastrectomy and neoadjuvant/ adjuvant treatment from 2002 to 2013, overall and subgroup by demographic characteristics and the receipt of radiation therapy

\begin{tabular}{|c|c|c|c|c|c|c|c|}
\hline & No. & $\begin{array}{l}\text { Cost of primary } \\
\text { treatment }(\$)^{a), b}\end{array}$ & p-value & $\begin{array}{l}\text { Net medical } \\
\text { cost }(\$)^{b), c}\end{array}$ & p-value & $\begin{array}{l}\text { 5-Year } \\
\text { OS (\%) }\end{array}$ & $\begin{array}{c}\text { 5-Year } \\
\text { DSS (\%) }\end{array}$ \\
\hline \multicolumn{8}{|l|}{ Sex } \\
\hline Male & 1,227 & $8,713 \pm 216$ & 0.339 & $16,097 \pm 406$ & 0.458 & 65.8 & 71.1 \\
\hline Female & 592 & $8,351 \pm 309$ & & $15,572 \pm 574$ & & 68.2 & 71.3 \\
\hline \multicolumn{8}{|l|}{ Age (yr) } \\
\hline 20-49 & 386 & $7,941 \pm 294$ & 0.263 & $16,175 \pm 678$ & 0.026 & 72.3 & 72.5 \\
\hline $50-59$ & 459 & $8,730 \pm 346$ & & $16,980 \pm 709$ & & 72.7 & 75.6 \\
\hline $60-69$ & 581 & $8,688 \pm 321$ & & $16,146 \pm 638$ & & 65.8 & 71.2 \\
\hline $70-89$ & 392 & $8,946 \pm 445$ & & $14,117 \pm 570$ & & 54.7 & 64.2 \\
\hline \multicolumn{8}{|l|}{ Surgery type } \\
\hline TG & 517 & $11,513 \pm 398$ & $<0.001$ & $20,868 \pm 699$ & $<0.001$ & 48.5 & 52.1 \\
\hline STG & 1,302 & $7,437 \pm 181$ & & $13,965 \pm 357$ & & 73.7 & 78.6 \\
\hline \multicolumn{8}{|c|}{ Charlson comorbidity index } \\
\hline $0-1$ & 1,139 & $7,575 \pm 187$ & $<0.001$ & $14,787 \pm 385$ & $<0.001$ & 67.5 & 69.9 \\
\hline 2 & 327 & $8,892 \pm 346$ & & $16,632 \pm 715$ & & 69.3 & 72.7 \\
\hline 3 & 144 & $11,193 \pm 890$ & & $18,792 \pm 1,426$ & & 67.6 & 80.3 \\
\hline$\geq 4$ & 209 & $12,416 \pm 812$ & & $19,418 \pm 1,350$ & & 55.1 & 71.9 \\
\hline \multicolumn{8}{|l|}{ Income } \\
\hline$\leq 20$ percentile & 277 & $8,621 \pm 438$ & 0.402 & $14,731 \pm 763$ & 0.040 & 64.7 & 68.3 \\
\hline 21-80 percentile & 995 & $8,398 \pm 217$ & & $15,600 \pm 429$ & & 68.4 & 73.1 \\
\hline$\geq 81$ percentile & 547 & $8,940 \pm 375$ & & $17,125 \pm 674$ & & 64.1 & 69.1 \\
\hline \multicolumn{8}{|c|}{ Health insurance type } \\
\hline NHI & 1,761 & $8,591 \pm 181$ & 0.893 & $16,029 \pm 339$ & 0.089 & 67.1 & 71.8 \\
\hline Medical aid & 58 & $8,727 \pm 766$ & & $12,814 \pm 1,421$ & & 48.5 & 48.5 \\
\hline \multicolumn{8}{|l|}{ Residential area } \\
\hline Metropolitan & 859 & $8,687 \pm 283$ & 0.622 & $16,089 \pm 479$ & 0.643 & 66.9 & 71.7 \\
\hline Non-metropolitan & 960 & $8,513 \pm 219$ & & $15,781 \pm 459$ & & 66.2 & 70.7 \\
\hline \multicolumn{8}{|l|}{ Hospital location } \\
\hline Metropolitan & 1,594 & $8,575 \pm 194$ & 0.697 & $15,770 \pm 344$ & 0.207 & 66.9 & 71.5 \\
\hline Nonmetropolitan & 225 & $8,742 \pm 382$ & & $17,045 \pm 1,120$ & & 63.9 & 68.4 \\
\hline \multicolumn{8}{|c|}{ Hospital volume (beds) } \\
\hline$<1,000$ & 765 & $8,892 \pm 256$ & 0.017 & $14,172 \pm 507$ & 0.034 & 64.6 & 69.6 \\
\hline $1,000-1,999$ & 707 & $7,978 \pm 260$ & & $13,978 \pm 519$ & & 71.2 & 75.1 \\
\hline$\geq 2,000$ & 347 & $9,201 \pm 506$ & & $15,111 \pm 801$ & & 61.4 & 66.4 \\
\hline \multicolumn{8}{|l|}{ Disabled status } \\
\hline No & 1,654 & $8,422 \pm 172$ & 0.041 & $15,858 \pm 346$ & 0.511 & 66.9 & 71.1 \\
\hline Yes & 165 & $10,327 \pm 908$ & & $16,617 \pm 1,146$ & & 62.9 & 71.5 \\
\hline \multicolumn{8}{|l|}{ RT use } \\
\hline No & 1,741 & $8,441 \pm 180$ & $<0.001$ & $15,642 \pm 337$ & $<0.001$ & 67.1 & 71.4 \\
\hline Yes & 78 & $12,175 \pm 792$ & & $22,526 \pm 1,623$ & & 58.0 & 68.7 \\
\hline \multicolumn{8}{|l|}{ Death during study } \\
\hline Yes & 607 & $11,234 \pm 422$ & $<0.001$ & $24,160 \pm 701$ & $<0.001$ & - & - \\
\hline No & 1,212 & $7,274 \pm 147$ & & $11,804+288$ & & - & - \\
\hline
\end{tabular}

OS, overall survival; DSS, disease-specific survival; TG, total gastrectomy; STG, subtotal gastrectomy; NHI, National Health Insurance; RT, radiotherapy. ${ }^{a}$ Defined as medical bill expenses claimed by medical service providers for primary treatment, ${ }^{b}$ All costs are presented in U.S. dollars, with an exchange rate of 1,103 Korean won to 1 U.S. dollar, which was the annual exchange rate in 2008 , ${ }^{\mathrm{c}}$ Defined as medical bill expenses claimed by medical service providers from diagnosis to death or last follow-up. 
71.1\% (95\% CI, 68.8 to 73.5). Compared with patients received chemotherapy alone, those who received CRT had inferior 5 -year OS $(\mathrm{p}=0.020)$ (Table 4). However, the 5-year DSS was similar according to the use of RT (no RT vs. RT; 71.4\% vs. $68.7 \%, \mathrm{p}=0.347$ ), which is understandable given the high proportion of comorbid patients in RT group. Being older $(\geq 60$ years), having a total gastrectomy, having a higher CCI (4), and receiving medical aid were significantly related to worse survival (S7 and S8 Tables).

\section{Discussion}

This study highlights the national patterns of neoadjuvant and adjuvant RT use for gastrectomy patients in Korea as well as the sociodemographic factors associated with its use. The results showed that RT usage was exceedingly low and primarily concentrated in one large-volume hospital. In comparison, there was a low rate of, but growing interest in, neoadjuvant chemotherapy (one-third of the hospitals in the nation). Adjuvant chemotherapy accounted for about $90 \%$ of the therapy modalities used and was mainstream in nearly all hospitals. These practice patterns are concerning given that RT, which has already been verified and accepted as an essential adjuvant therapy modality for gastric cancer in the Unites States [18], has not had enough opportunity to develop the evidence in D2-dissected patients.

We found that only $4 \%$ of patients received RT as an adjuvant or neoadjuvant treatment. Furthermore, our results contradict the results of the previous Surveillance, Epidemiology, and End Results studies [19]. For example, in the United States, the proportion of patients who underwent surgery alone after 2000 decreased by at least $13 \%$, but the proportion of patients who underwent CRT increased from $8 \%-14 \%$ to $25 \%-33 \%$. This implies that, in Korea, the results of the INT-0116 trial, unlike in the United States [19,20], did not affect treatment practices.

The discrepancy between patterns and trends in the United States and Korea may be explained by the extent and safety of lymphadenectomies performed in East Asia [21]. According to the 2004 the Korean Gastric Cancer Association (KGCA) nationwide survey, the quality of extensive D2 lymphadenectomy, represented by the number of retrieved lymph nodes, was assured in most of the 57 hospitals belonging to the KGCA regardless of hospital volume [22]. In contrast to the high postoperative mortality rate $(\sim 10 \%)$ in trials conducted in Western countries [23-25], the very low mortality rate $(<1 \%)$ for D2 dissection observed in prior Phase III trials in Korea [12] and Japan [26] was reaffirmed on a population level in the present study $(0.6 \%)$. Although we found that patients who were $\geq 70$ years old, had a high comorbidity burden $(\mathrm{CCI} \geq 4)$, and who had a more severe cancer stage (having undergone total gastrectomy) were associated significantly, we found only a slight increase in the risk of postoperative mortality.

In addition, our data showed that the majority of RT patients were concentrated in one large-volume hospital [8]. The proportion of RT administered in this hospital was greater in magnitude (52\%) than the proportion of RT administered in all other 25 hospitals combined. Otherwise, RT was more likely to be utilized in small- or medium-volume hospitals located in nonmetropolitan areas. Although we were not able to investigate whether tumor factors, such as cancer stage or adverse pathologic features, influenced the consideration of RT, we found significant interhospital variations (Fig. 1A). This indicates that cultural predispositions rather than universal criteria guide clinical decisions.

Furthermore, being over 60 years old and having a low income ( $\leq 20$ th percentile) were barriers to receiving RT. On the other hand, we found that patients with a high comorbidity burden were more likely to receive RT. This is most likely because trimodal therapy including RT is used for patients who may be intolerant to full-dose chemotherapy, such as those with a high comorbidity burden. In our opinion, studies are needed to determine the role of RT in the clinical setting among patients that might be intolerant to full-dose chemotherapy.

Moreover, the absolute proportion of patients receiving adjuvant chemotherapy ranged $83.2 \%-89.1 \%$. Furthermore, the 5-year OS and the 5-year DSS outcomes in this study $(66.5 \%$ and $71.5 \%$, respectively) were consistent or lower when compared to the Capecitabine and Oxaliplatin Adjuvant Study in Stomach Cancer (CLASSIC) [12] and the Adjuvant Chemotherapy Trial of TS-1 for Gastric Cancer (ACTS-GC) [11] trials; however, these two trials included younger patients with fewer comorbidities than observed in the general population, which may account for any discrepancies. The overwhelming use of adjuvant chemotherapy found in our study might be rooted, to some degree, in a propensity for clinicians to depend heavily on evidence generated from our country. Nevertheless, this consistent usage, which existed before 2007, is worrisome because it may indicate that these practices were determined before maturation of emerging evidence.

The addition of RT to adjuvant/neoadjuvant chemotherapy increased the mean cost of $\$ 3,734$ in Korea that has single-payer health care system. We could not find that addition of RT reduced the net medical cost during follow-up that indirectly reflected recurrence preventing treatment effects. In addition, it was found that RT did not increase the 5-year DSS rate (RT vs. no RT, $68.7 \%$ vs. $71.4 \%$ ), yet consistent with previous findings of ARTIST trial (5-year OS, 75\% vs. 73\%) 
and CRITICS trial (5-year OS, $40.9 \%$ vs. $41.3 \%$ ). Although we felt that use of RT in (neo-)/adjuvant setting (before)/after D2 surgery increased the total medical cost but not the survival rate in these general population, such a precondition would require (1) that the clinical indication was not different between patients with CRT and those with chemotherapy alone, and (2) that all other confounders, which were not identified in this study, were well balanced. Although previous reports imply the fulfillment of preconditions [27], we cannot justify these findings without details including tumor stage or residual tumor burden, but may question the importance and necessity of RT in D2-dissected patients [28]. However, several studies have demonstrated loco-regional recurrence usually occurs in the nodal basin outside the D2 dissection surgical field $[28,29]$ and a considerable rate of locoregional recurrence is observed in high-risk patients (e.g., $\mathrm{N}+$ ) even after $\mathrm{D} 2$ surgery [30]. In the ARTIST trial, there was a significant benefit in a subgroup of patients with $\mathrm{N}+$ with postoperative CRT. A subsequent ARTIST II trial is currently underway to refine the role of RT in patients with positive lymph node metastasis.

We also found that sociodemographic factors such as being $\geq 60$ years old, having a high comorbidity burden, and receiving medical aid were associated with poorer survival outcomes. Therefore, when making treatment decisions for individual patients, physicians should consider sociodemographic factors.

The current study has some limitations. For example, the NHIS-NSC database lacks information on gastric cancer staging and recurrence; therefore, the analyses of stage-specific patterns of care and the impact of neoadjuvant and adjuvant treatment on survival were limited. In our study, standardized incidences were not calculated, as the Korean Statistical Information Service do not provide the incidence information of the intersection of two sets (diagnosed as gastric cancer and underwent gastrectomy). Since our findings were computed in the crude rates which result from the specific composition which prevails in our sampled population, we caution against direct comparing other study populations with respect to actual numerical values. We also lacked direct clinical data regarding pathologic factors (such as residual tumor burden), radiation specifics (e.g., dose, fractionation, and field), performance status, and treatment compliance. Information on chemotherapy regimen was also unavailable for some patients, further limiting the analyses of chemotherapy patterns of care. Therefore, further studies examining the impact of RT on survival in well-designed prospective trials as well as in the general population are necessary. However, despite these limitations, this study also has a strength that the NHIS-NSC database represents nearly all patients in Korea, allowing us to generalize to the entire population in Korea.

The present study is the first large study to analyze the use of RT in the neoadjuvant and adjuvant management of gastric cancer in East Asia. We observed that RT was uncommonly utilized and that RT patients were concentrated heavily in one hospital. Considering the sizable cost overrun and lack of survival advantage for RT in general population, better risk stratifying D2-dissected patients is utterly critical to refine the role of RT in D2-dissected patients. It is important to recognize the cultural predispositions highlighted in our study since they might continue to influence physician practices and attitudes toward RT, despite emerging evidence.

\section{Electronic Supplementary Material}

Supplementary materials are available at Cancer Research and Treatment website (http://www.e-crt.org).

\section{Conflicts of Interest}

Conflict of interest relevant to this article was not reported.

\section{Acknwledgments}

The authors thank Mr. Dong-Su Jang, Research Assistant, Department of Anatomy, Yonsei University College of Medicine, Seoul, South Korea, for his help with the figure. Supported by a faculty research grant from Yonsei University College of Medicine (Grant No. 6-2015-0037, Woong Sub Koom) and by the National Research Foundation of Korea (NRF) grant funded by the Korea government (MSIP) (2015R1C1A2A01055617, Hyo Song Kim).

\section{References}

1. Torre LA, Bray F, Siegel RL, Ferlay J, Lortet-Tieulent J, Jemal A. Global cancer statistics, 2012. CA Cancer J Clin. 2015;65: 87-108.

2. Colquhoun A, Arnold M, Ferlay J, Goodman KJ, Forman D,
Soerjomataram I. Global patterns of cardia and non-cardia gastric cancer incidence in 2012. Gut. 2015;64:1881-8.

3. Jung KW, Won YJ, Kong HJ, Oh CM, Cho H, Lee DH, et al. Cancer statistics in Korea: incidence, mortality, survival, and 
prevalence in 2012. Cancer Res Treat. 2015;47:127-41.

4. Lim DH, Kim DY, Kang MK, Kim YI, Kang WK, Park CK, et al. Patterns of failure in gastric carcinoma after D2 gastrectomy and chemoradiotherapy: a radiation oncologist's view. Br J Cancer. 2004;91:11-7.

5. Knight G, Earle CC, Cosby R, Coburn N, Youssef Y, Malthaner $\mathrm{R}$, et al. Neoadjuvant or adjuvant therapy for resectable gastric cancer: a systematic review and practice guideline for North America. Gastric Cancer. 2013;16:28-40.

6. Fujitani K. Overview of adjuvant and neoadjuvant therapy for resectable gastric cancer in the East. Dig Surg. 2013;30:119-29.

7. Macdonald JS, Smalley SR, Benedetti J, Hundahl SA, Estes NC, Stemmermann GN, et al. Chemoradiotherapy after surgery compared with surgery alone for adenocarcinoma of the stomach or gastroesophageal junction. N Engl J Med. 2001;345: 725-30.

8. Lee J, Lim DH, Kim S, Park SH, Park JO, Park YS, et al. Phase III trial comparing capecitabine plus cisplatin versus capecitabine plus cisplatin with concurrent capecitabine radiotherapy in completely resected gastric cancer with D2 lymph node dissection: the ARTIST trial. J Clin Oncol. 2012;30:268-73.

9. Verheij M, Jansen EP, Cats A, van Grieken NC, Aaronson NK, Boot $\mathrm{H}$, et al. A multicenter randomized phase III trial of neoadjuvant chemotherapy followed by surgery and chemotherapy or by surgery and chemoradiotherapy in resectable gastric cancer: first results from the CRITICS study. J Clin Oncol. 2016;34(15 Suppl):Abstr 4000.

10. Kim TH, Park SR, Ryu KW, Kim YW, Bae JM, Lee JH, et al. Phase 3 trial of postoperative chemotherapy alone versus chemoradiation therapy in stage III-IV gastric cancer treated with R0 gastrectomy and D2 lymph node dissection. Int J Radiat Oncol Biol Phys. 2012;84:e585-92.

11. Sakuramoto S, Sasako M, Yamaguchi T, Kinoshita T, Fujii M, Nashimoto A, et al. Adjuvant chemotherapy for gastric cancer with S-1, an oral fluoropyrimidine. N Engl J Med. 2007;357: 1810-20.

12. Bang YJ, Kim YW, Yang HK, Chung HC, Park YK, Lee KH, et al. Adjuvant capecitabine and oxaliplatin for gastric cancer after D2 gastrectomy (CLASSIC): a phase 3 open-label, randomised controlled trial. Lancet. 2012;379:315-21.

13. Schmidt B, Yoon SS. D1 versus D2 lymphadenectomy for gastric cancer. J Surg Oncol. 2013;107:259-64.

14. Schwarz RE, Smith DD. Clinical impact of lymphadenectomy extent in resectable gastric cancer of advanced stage. Ann Surg Oncol. 2007; 14:317-28.

15. Lee J, Lee JS, Park SH, Shin SA, Kim K. Cohort Profile: The National Health Insurance Service-National Sample Cohort (NHIS-NSC), South Korea. Int J Epidemiol. 2017;46:e15.

16. Charlson ME, Pompei P, Ales KL, MacKenzie CR. A new method of classifying prognostic comorbidity in longitudinal studies: development and validation. J Chronic Dis. 1987;40: 373-83.

17. Choi KS, Kwak MS, Lee HY, Jun JK, Hahm MI, Park EC. Screening for gastric cancer in Korea: population-based preferences for endoscopy versus upper gastrointestinal series. Cancer Epidemiol Biomarkers Prev. 2009;18:1390-8.
18. Smalley SR, Benedetti JK, Haller DG, Hundahl SA, Estes NC, Ajani JA, et al. Updated analysis of SWOG-directed intergroup study 0116: a phase III trial of adjuvant radiochemotherapy versus observation after curative gastric cancer resection. J Clin Oncol. 2012;30:2327-33.

19. Seyedin S, Wang PC, Zhang Q, Lee P. Benefit of adjuvant chemoradiotherapy for gastric adenocarcinoma: a SEER population analysis. Gastrointest Cancer Res. 2014;7:82-90.

20. Cheng J, Squires MH 3rd, Mikell JL, Fisher SB, Staley CA 3rd, Kooby DA, et al. Radiotherapy patterns of care in gastric adenocarcinoma: a single institution experience. J Gastrointest Oncol. 2015;6:247-53.

21. Ashraf N, Hoffe S, Kim R. Adjuvant treatment for gastric cancer: chemotherapy versus radiation. Oncologist. 2013;18: 1013-21.

22. The Information Committee of the Korean Gastric Cancer Association. 2004 Nationwide gastric cancer report in Korea. J Korean Gastric Cancer Assoc. 2007;7:47-54.

23. Cuschieri A, Weeden S, Fielding J, Bancewicz J, Craven J, Joypaul V, et al. Patient survival after D1 and D2 resections for gastric cancer: long-term results of the MRC randomized surgical trial. Surgical Co-operative Group. Br J Cancer. 1999;79: 1522-30.

24. Hartgrink HH, van de Velde CJ, Putter H, Bonenkamp JJ, Klein Kranenbarg E, Songun I, et al. Extended lymph node dissection for gastric cancer: who may benefit? Final results of the randomized Dutch gastric cancer group trial. J Clin Oncol. 2004;22:2069-77.

25. Bonenkamp JJ, Hermans J, Sasako M, van de Velde CJ, Welvaart K, Songun I, et al. Extended lymph-node dissection for gastric cancer. N Engl J Med. 1999;340:908-14.

26. Sano T, Sasako M, Yamamoto S, Nashimoto A, Kurita A, Hiratsuka M, et al. Gastric cancer surgery: morbidity and mortality results from a prospective randomized controlled trial comparing D2 and extended para-aortic lymphadenectomy: Japan Clinical Oncology Group study 9501. J Clin Oncol. 2004;22:2767-73.

27. Kim S, Lim DH, Lee J, Kang WK, MacDonald JS, Park CH, et al. An observational study suggesting clinical benefit for adjuvant postoperative chemoradiation in a population of over 500 cases after gastric resection with D2 nodal dissection for adenocarcinoma of the stomach. Int J Radiat Oncol Biol Phys. 2005;63:1279-85.

28. Chang JS, Koom WS, Lee Y, Yoon HI, Lee HS. Postoperative adjuvant chemoradiotherapy in D2-dissected gastric cancer: is radiotherapy necessary after D2-dissection? World J Gastroenterol. 2014;20:12900-7.

29. Chang JS, Lim JS, Noh SH, Hyung WJ, An JY, Lee YC, et al. Patterns of regional recurrence after curative D2 resection for stage III (N3) gastric cancer: implications for postoperative radiotherapy. Radiother Oncol. 2012;104:367-73.

30. Yu JI, Lim do H, Ahn YC, Lee J, Kang WK, Park SH, et al. Effects of adjuvant radiotherapy on completely resected gastric cancer: a radiation oncologist's view of the ARTIST randomized phase III trial. Radiother Oncol. 2015;117:171-7. 